\title{
HAT-P-31b,c: A TRANSITING, ECCENTRIC, HOT JUPITER AND A LONG-PERIOD, MASSIVE THIRD BODY*
}

\author{
D. M. Kipping ${ }^{1,2}$, J. Hartman ${ }^{1}$, G. Á. Bakos ${ }^{1}$, G. Torres ${ }^{1}$, D. W. LAtham ${ }^{1}$, D. Bayliss ${ }^{3}$, L. L. Kiss ${ }^{3}$, B. Sato ${ }^{4}$, B. Béky ${ }^{1}$, \\ Géza Kovács $^{5}$, S. N. Quinn ${ }^{1}$, L. A. Buchhave ${ }^{6}$, J. Andersen ${ }^{6}$, G. W. Marcy ${ }^{7}$, A. W. Howard ${ }^{7}$, D. A. Fischer $^{8}$, \\ J. A. Johnson ${ }^{9}$, R. W. Noyes ${ }^{1}$, D. D. SAsselov ${ }^{1}$, R. P. StefaniK ${ }^{1}$, J. LÁzár ${ }^{10}$, I. PAPp ${ }^{10}$, P. SÁri ${ }^{10}$, And G. Fúrész ${ }^{1}$ \\ ${ }^{1}$ Harvard-Smithsonian Center for Astrophysics, Cambridge, MA, USA; dkipping@cfa.harvard.edu \\ ${ }^{2}$ Department of Physics \& Astronomy, University College London, London, UK \\ ${ }^{3}$ Research School of Astronomy and Astrophysics, The Australian National University, Weston Creek, ACT, Australia \\ ${ }^{4}$ Department of Earth and Planetary Sciences, Tokyo Institute of Technology, 2-12-1 Ookayama, Meguro-ku, Tokyo 152-8551, Japan \\ ${ }^{5}$ Konkoly Observatory, Budapest, Hungary \\ ${ }^{6}$ Niels Bohr Institute, Copenhagen University, Copenhagen, Denmark \\ ${ }^{7}$ Department of Astronomy, University of California, Berkeley, CA \\ ${ }^{8}$ Department of Astronomy, Yale University, New Haven, CT, USA \\ ${ }^{9}$ Department of Astrophysics, California Institute of Technology, Pasadena, CA, USA \\ ${ }^{10}$ Hungarian Astronomical Association, Budapest, Hungary \\ Received 2011 June 6; accepted 2011 July 2; published 2011 August 16
}

\begin{abstract}
We report the discovery of HAT-P-31b, a transiting exoplanet orbiting the $V=11.660$ dwarf star GSC 209900908. HAT-P-31b is the first planet discovered with the Hungarian-made Automated Telescope (HAT) without any follow-up photometry, demonstrating the feasibility of a new mode of operation for the HATNet project. The $2.17 M_{J}, 1.1 R_{J}$ planet has a period of $P_{b}=5.0054$ days and maintains an unusually high eccentricity of $e_{b}=0.2450 \pm 0.0045$, determined through Keck, FIbr-fed Échelle Spectrograph, and Subaru high-precision radial velocities (RVs). Detailed modeling of the RVs indicates an additional quadratic residual trend in the data detected to very high confidence. We interpret this trend as a long-period outer companion, HAT-P-31c, of minimum mass $3.4 M_{J}$ and period $\geqslant 2.8$ years. Since current RVs span less than half an orbital period, we are unable to determine the properties of HAT-P-31c to high confidence. However, dynamical simulations of two possible configurations show that orbital stability is to be expected. Further, if HAT-P-31c has non-zero eccentricity, our simulations show that the eccentricity of HAT-P-31b is actively driven by the presence of c, making HAT-P-31 a potentially intriguing dynamical laboratory.
\end{abstract}

Key words: planetary systems - stars: individual (HAT-P-31, GSC 2099-00908) - techniques: photometric techniques: spectroscopic

Online-only material: machine-readable and VO tables

\section{INTRODUCTION}

Transiting extrasolar planets provide invaluable insight into the nature of planetary systems. The opportunities for follow-up include spectroscopic inference of an exoplanet's atmosphere (Tinetti et al. 2007), searches for dynamical variations (Agol et al. 2005; Kipping 2009a, 2009b), and characterization of the orbital elements (Winn et al. 2011). Multi-planet systems in particular offer rich dynamical interactions and their frequency is key to understanding planet formation.

The Hungarian-made Automated Telescope Network (HATNet; Bakos et al. 2004) survey for transiting exoplanets (TEPs) around bright stars operates six wide-field instruments: four at the Fred Lawrence Whipple Observatory (FLWO) in Arizona and two on the roof of the hangar servicing the Smithsonian Astrophysical Observatory's Submillimeter Array, in Hawaii. Since 2006, HATNet has announced and published 30 TEPs (e.g., Johnson et al. 2011). In this work, we report

\footnotetext{
* Based in part on observations obtained at the W. M. Keck Observatory, which is operated by the University of California and the California Institute of Technology. Keck time has been granted by NASA (N167Hr). Based in part on data collected at Subaru Telescope, which is operated by the National Astronomical Observatory of Japan. Based in part on observations made with the Nordic Optical Telescope, operated on the island of La Palma jointly by Denmark, Finland, Iceland, Norway, and Sweden, in the Spanish Observatorio del Roque de los Muchachos of the Instituto de Astrofisica de Canarias.
}

our thirty-first discovery, around the relatively bright star GSC 2099-00908. In addition, a long-period companion is detected through detailed modeling of the radial velocities (RVs), although no transits of this object have been detected or are necessarily expected.

In Section 2, we summarize the detection of the photometric transit signal and the subsequent spectroscopic observations of HAT-P-31 to confirm the planet. In Section 3, we analyze the data to determine the stellar and planetary parameters. Our findings are discussed in Section 4.

\section{OBSERVATIONS}

As described in detail in several previous papers (e.g., Bakos et al. 2010; Latham et al. 2009), HATNet employs the following methods to discover transiting planets: (1) identification of candidate transiting planets based on HATNet photometric observations, (2) high-resolution, low signal-to-noise $(\mathrm{S} / \mathrm{N})$ "reconnaissance" spectra to efficiently reject many false positives, (3) higher-precision photometric observations during transit to refine transit parameters and obtain the light curve derived stellar density, and (4) high-resolution, high-S/N "confirmation" spectroscopy to detect the orbital motion of the star due to the planet, characterize the host star, and rule out blend scenarios. 
In this work, step (3) is omitted and this is the biggest difference from the usual HATNet analysis. The detection, and thus verification, of an exoplanet can be made using step (4) alone. Indeed, the majority of exoplanets have been found in this way. Step (1) clearly allows us to intelligently select the most favorable targets for this resource-intensive activity though. Step (2) follows the same logic. Step (3) is predominantly for the purposes of characterizing the system, and therefore its omission does not impinge on the planet detection. In some cases, followup photometry is used to confirm marginal HATNet candidate detections as well, but this is not the case for the discovery presented in this work. An additional check on step (4) is that the derived ephemeris is consistent with that determined photometrically.

We did not obtain high-precision photometry for this target as the transits were not observable from our usual site of choice, FLWO, until at least 2012 May. This is because the transiting planet has a near-integer period and the time of transit minimum has now phased into the day-light hours in Arizona (i.e., unobservable). Rather than wait until this time, we have decided to release this confirmed planet detection to the community so that follow-up photometry may be conducted at other sites.

The principal consequence of not having any follow-up photometry is that the obtainable precision of the transit parameters is reduced. In the case of HAT-P-31b, this means that the light curve derived density was less precise than that determined spectroscopically. In practice then, we reverse the usual logic and instead of applying a prior on the stellar density from the light curve, we apply a prior on the light curve from the stellar density. One can see that the decision on this will vary from case to case depending upon the transit depth and target brightness.

Another issue is that in the past HAT analyses have used the HATNet photometry for measuring the planetary ephemeris, $P$ and $\tau$, and little else. All other parameters could be more precisely determined from the follow-up photometry. As a consequence, we used the External Parameter Decorrelation (EPD; see Bakos et al. 2010) and Trend Filtering Algorithm (TFA; see Kovács et al. 2005) techniques to correct the HATNet photometry, which are known to attenuate the apparent transit depth by a small amount. This was usually accounted for by including an instrumental blending factor, $B_{\text {inst }}$, in the HATNet data, which could be determined by comparing the ratio of the HATNet apparent depth and the follow-up photometry depth. Without follow-up photometry, $B_{\text {inst }}$ would be unconstrained and so an estimation of the planetary radius would be impossible. To avoid this, we employ the more computationally demanding and sophisticated technique of reconstructive TFA (Kovács et al. 2005). Reconstructive TFA does not attenuate the transit depth significantly and thus offers a way of avoiding a free $B_{\text {inst }}$ term. Our previous experience with the two modes of TFA supports this. For example, HAT-P-15b's TFA photometry (Kovács et al. 2010) was found to require a blending factor of $B_{\text {inst }}=0.71 \pm 0.07$. In contrast, the reconstructive TFA for the same planet causes $B_{\text {inst }}=0.95 \pm 0.04$. We find no instance in any previous implementation of reconstructive TFA where $B_{\text {inst }}$ would depart from unity by more than $2 \sigma$. We will therefore use the reconstructive TFA in this work and conservatively double all uncertainties relating to the depth and radius of the planet.

In this paper, we will consequently show that HATNet photometry alone is sufficient to constrain the system properties

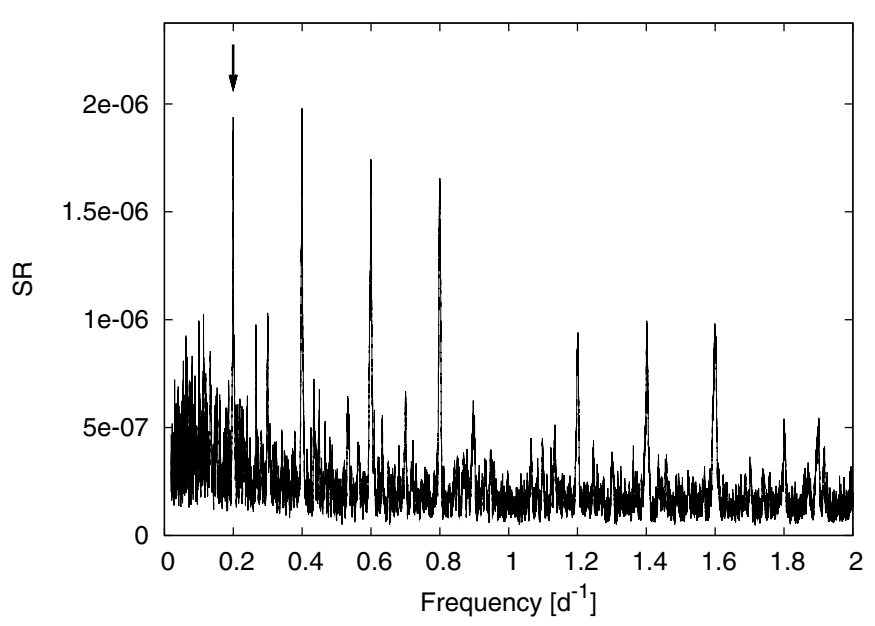

Figure 1. BLS (Kovács et al. 2002) periodogram of HATNet photometry. The arrow marks the true transit signal and the other peaks are interpreted to be aliases. The $y$-axis denotes signal residue (SR); see Kovács et al. (2002) for details of the definition.

and that future work may not always require step (3) (i.e., followup photometry).

In the following subsections we highlight specific details of this procedure that are pertinent to the discovery of HAT-P-31b.

\subsection{Photometric Detection}

The transits of HAT-P-31b were photometrically detected with a combined confidence of $6.2 \sigma$ using the HAT-5 telescope in Arizona and the HAT- 8 telescope in Hawaii. The region around GSC 2099-00908, a field internally labeled as 241, was observed between 2007 March and 2007 July, whenever weather conditions permitted. In total, we gathered 9205 exposures of 5 minutes at a 5.5 minute cadence. Of these, 769 images were rejected by our reduction pipeline because they produced bad photometry for a significant fraction of stars. A typical image is found to contain approximately 48,000 stars down to $I_{C} \sim 14$. For the brightest stars in the field, the photometric precision per-image was $3 \mathrm{mmag}$.

Standard photometric procedures were used to calibrate the HATNet frames and then these calibrated images were subjected to star detection and astrometry, as described in Pál \& Bakos (2006). Aperture photometry was performed on each image at the stellar centroids derived from the Two Micron All Sky Survey (2MASS; Skrutskie et al. 2006) catalog and the individual astrometric solutions. The resulting light curves were decorrelated (cleaned of trends) using the EPD (see Bakos et al. 2010) technique in "constant" mode and TFA (see Kovács et al. 2005).

The light curves were searched for transits using the Boxfitting Least-Squares (BLS; Kovács et al. 2002) method. We detected a significant signal in the light curve of GSC 209900908 (also known as 2MASS 18060904+2625359; $\alpha=$ $18^{\mathrm{h}} 06^{\mathrm{m}} 09^{\mathrm{s}} .00, \delta=+26^{\circ} 25^{\prime} 36^{\prime \prime}$. 0 ; J2000; $V=11.660$; Droege et al. 2006), with an apparent depth of $\sim 5.1 \mathrm{mmag}$, and a period of $P=5.0050$ days. The BLS periodogram is shown in Figure 1. The drop in brightness had a first-to-last-contact duration, relative to the total period, of $q=0.0432 \pm 0.0038$, 
Table 1

HATNet Differential Photometry of HAT-P-31

\begin{tabular}{cccc}
\hline \hline $\begin{array}{l}\text { BJD } \\
(2,400,000+)\end{array}$ & ${\text { Mag }(\text { EPD })^{\mathrm{a}}}$ & ${\operatorname{Mag}(\mathrm{TFA})^{\mathrm{b}}}$ & $\sigma_{\text {Mag }}$ \\
\hline 54178.0007000 & 11.4164 & 11.4203 & 0.0075 \\
54178.0045362 & 11.4267 & 11.4273 & 0.0073 \\
54178.0083829 & 11.4250 & 11.4317 & 0.0075 \\
54178.0122220 & 11.4177 & 11.4275 & 0.0069 \\
54178.0160619 & 11.4244 & 11.4220 & 0.0072 \\
$:$ & $:$ & $\vdots$ & $:$ \\
\hline
\end{tabular}

Notes.

a These magnitudes have been subjected to the EPD procedure.

$\mathrm{b}$ These magnitudes have been subjected to the EPD and TFA procedures.

(This table is available in its entirety in machine-readable and Virtual Observatory (VO) forms in the online journal. A portion is shown here for guidance regarding its form and content.)

corresponding to a total duration of $5.187 \pm 0.462 \mathrm{hr}$. Due to the lack of follow-up photometry, the HATNet photometry was reprocessed with more computationally expensive reconstructive TFA, as discussed in Section 2 and shown in Figure 2. The EPD and reconstructive TFA corrected photometry is provided in Table 1.

\subsection{Reconnaissance Spectroscopy}

High-resolution, low-S/N reconnaissance spectra were obtained for HAT-P-31 using the Tillinghast Reflector Echelle Spectrograph (TRES; Fúrész et al. 2008) on the $1.5 \mathrm{~m}$ Tillinghast Reflector at FLWO, and the echelle spectrograph on the Australian National University (ANU) 2.3 m telescope at Siding Spring Observatory in Australia. The two TRES spectra of HAT-P-31 were obtained, reduced, and analyzed to measure the stellar effective temperature, surface gravity, projected rotation velocity, and RV via cross-correlation against a library of synthetic template spectra. The reduction and analysis procedure has been described by Quinn et al. (2010) and Buchhave et al. (2010). A total of 14 spectra of HAT-P-31 were obtained with the ANU $2.3 \mathrm{~m}$ telescope. These data were collected, reduced, and analyzed to measure the RV via cross-correlation against the spectrum of an RV standard star HD 223311 following the procedure described by Béky et al. (2011). The resulting measurements from TRES and the ANU $2.3 \mathrm{~m}$ telescope are given in Table 2.
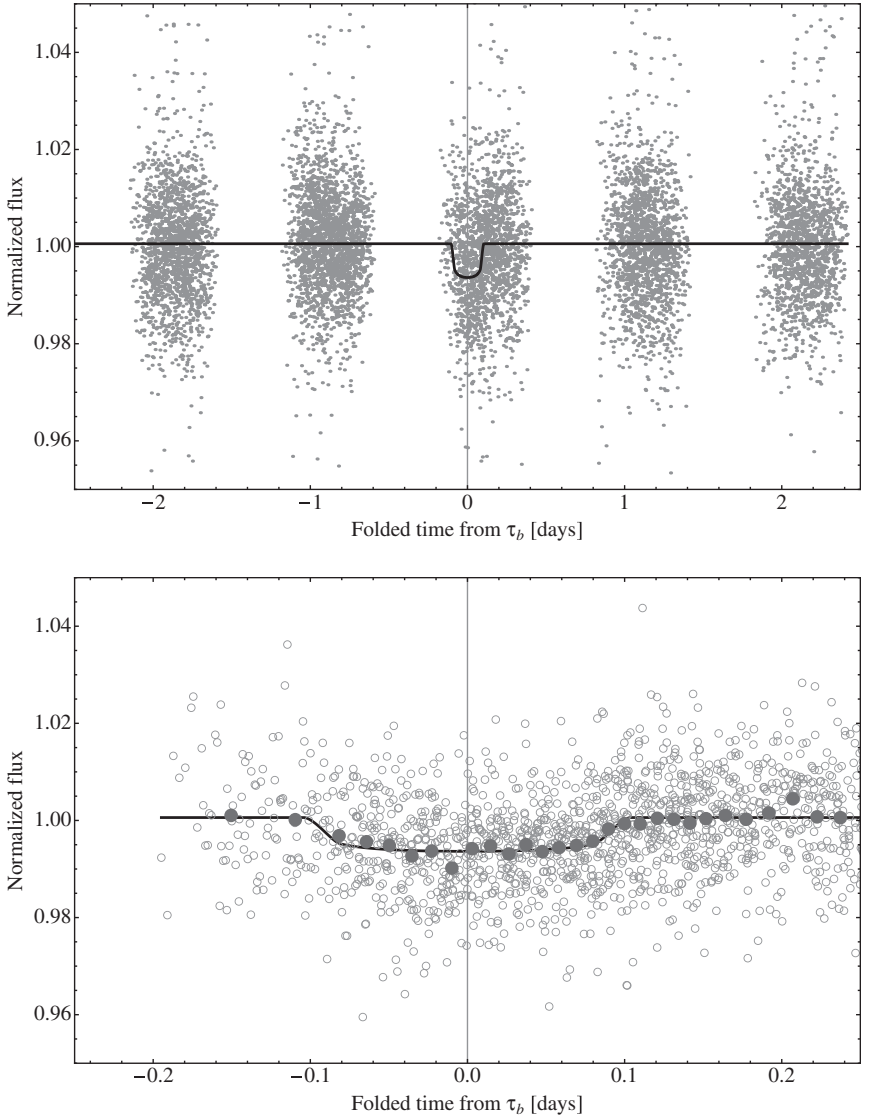

Figure 2. Unbinned light curve of HAT-P-31 including all 8436 instrumental $I_{C}$ band 5.5 minute cadence measurements obtained with the HAT-5 and HAT-8 telescopes of HATNet (see the text for details), and folded with the period $P=5.005425$ days resulting from the global fit described in Section 3 . The solid line shows our transit model fit to the light curve (Section 3.2). The bottom panel shows a zoomed-in view of the transit, the filled circles show the light curve binned in phase with a bin size of 50 points.

These observations revealed no detectable RV variation at the $1 \mathrm{~km} \mathrm{~s}^{-1}$ precision of the observations. Additionally the spectra are consistent with a single, slowly rotating, dwarf star.

\subsection{High-resolution, High-S/N Spectroscopy}

We proceeded with the follow-up of this candidate by obtaining high-resolution, high-S/N spectra to characterize the $\mathrm{RV}$ variations and to refine the determination of the stellar

Table 2

Summary of Reconnaissance Spectroscopy Observations of HAT-P-31

\begin{tabular}{lcccccr}
\hline \hline Instrument & Date & $\begin{array}{c}\text { Number of } \\
\text { Spectra }\end{array}$ & $\begin{array}{c}T_{\text {eff }} \\
(\mathrm{K})\end{array}$ & $\log \left(g_{*}(\mathrm{cgs})\right)$ & $\begin{array}{c}v \sin i \\
\left(\mathrm{~km} \mathrm{~s}^{-1}\right)\end{array}$ \\
\hline TRES & 2009 Jul 5 & 1 & 6000 & 4.0 & 4 & $\begin{array}{c}\gamma_{\mathrm{RV}}^{\mathrm{a}} \\
\left(\mathrm{km} \mathrm{s}^{-1}\right)\end{array}$ \\
TRES & 2009 Jul 7 & 1 & 6000 & 4.0 & 4 & -2.342 \\
ANU 2.3 m & 2009 Jul 14 & 5 & $\ldots$ & $\ldots$ & $\ldots$ & $-8.07 \pm 0.35$ \\
ANU 2.3 m & 2009 Jul 17 & 5 & $\ldots$ & $\ldots$ & $\ldots$ & $-7.13 \pm 0.40$ \\
ANU 2.3 m & 2009 Jul 18 & 2 & $\ldots$ & $\ldots$ & $\ldots$ & $-7.64 \pm 0.44$ \\
ANU 2.3 m & 2009 Jul 19 & 2 & $\ldots$ & $\ldots .54 \pm 0.49$ \\
\hline
\end{tabular}

Notes. ${ }^{\text {a }}$ The mean heliocentric RV of the target. Systematic differences between the velocities from the two instruments are consistent with the velocity zero-point uncertainties. For the ANU $2.3 \mathrm{~m}$ observations we give the weighted mean of the observations and the uncertainty on the mean for each night. Note that the systematic difference of $-5.3 \mathrm{~km} \mathrm{~s}^{-1}$ between the ANU $2.3 \mathrm{~m}$ and TRES observations is similar to the difference of $-5.1 \mathrm{~km} \mathrm{~s}^{-1}$ found between these same two instruments by Béky et al. (2011) for HAT-P-27. 

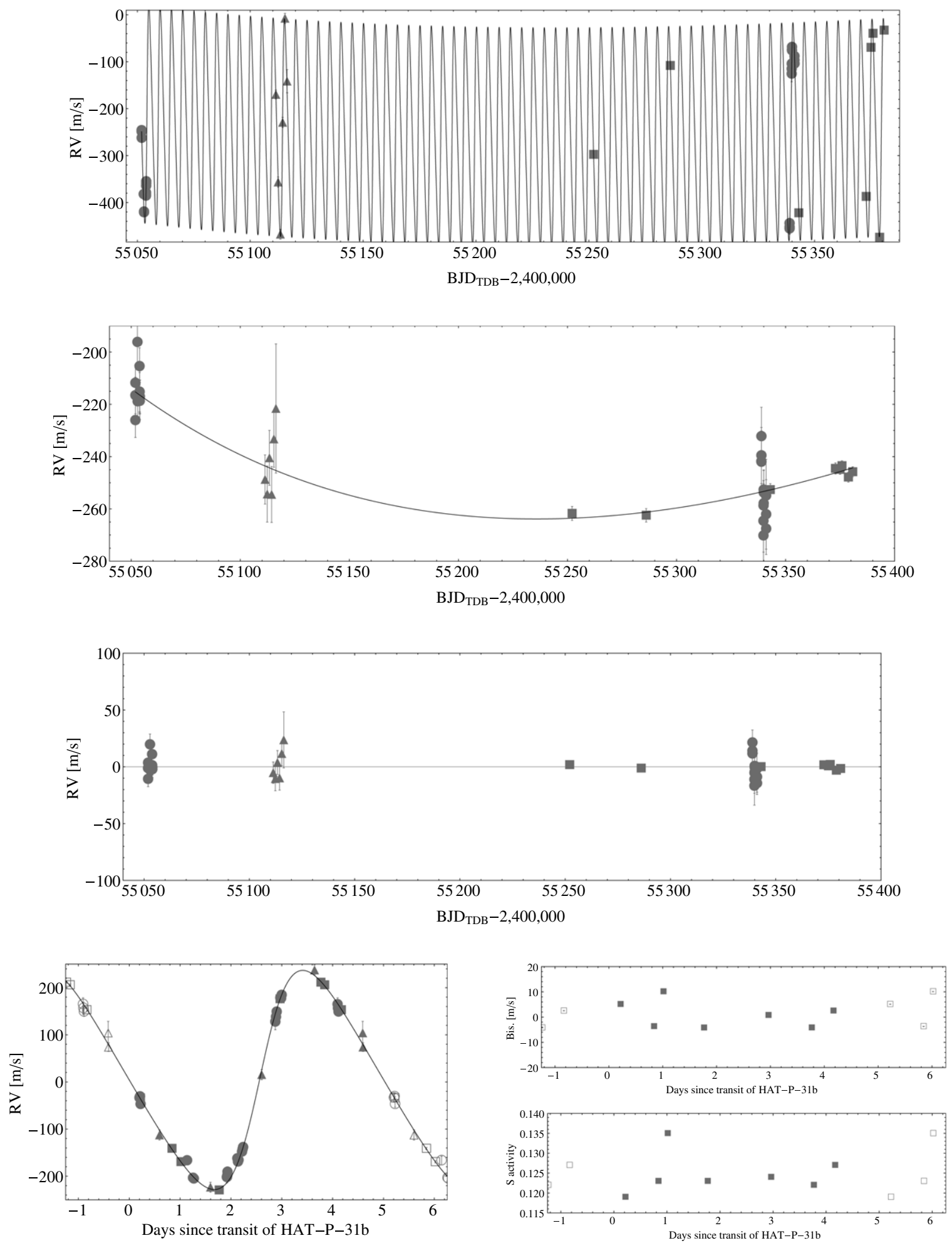

Figure 3. First row: Keck/HIRES (squares), Subaru (circles), and FIES (triangles) RV measurements for HAT-P-31, along with our best-fit two-planet model (see Table 7). The center-of-mass velocity has been subtracted. Second row: same as the top panel except the RV model of the inner planet has been subtracted from the data and the model, revealing the orbit of the outer planet. The $\chi^{2}$ of the best-fit two-planet model is 34.8 for 39 data points $\left(\mathrm{rms}\right.$ of $9.60 \mathrm{~m} \mathrm{~s}^{-1}$ ) indicating a stellar jitter at or below the measurements errors. Third row: residuals from our best-fit model. Lower left: RV measurements phased to the orbital periods of the inner planet (left). The quadratic trend has been removed. Lower right: upper panel shows the bisector spans (BS), with the mean value subtracted, phased at the period of the inner planet. Lower panel shows relative chromospheric activity index $S$ measured from the Keck spectra, phased at the period of the inner planet. Note the different vertical scales of the panels. Observations shown twice are represented with open symbols.

parameters. For this we used the High Resolution Echelle Spectrometer (HIRES) instrument (Vogt et al. 1994) with the iodine-cell (Marcy \& Butler 1992) on the Keck I telescope, the High-Dispersion Spectrograph (HDS; Noguchi et al. 2002) with the iodine-cell (Sato et al. 2002) on the Subaru telescope, and the FIbr-fed Échelle Spectrograph (FIES) on the $2.5 \mathrm{~m}$ Nordic Optical Telescope (Djupvik \& Andersen 2010). Table 3 summarizes the observations. The table also provides references 
Table 3

Summary of High-resolution, High-S/N

Spectroscopy Observations of HAT-P-31

\begin{tabular}{llcc}
\hline \hline Instrument & \multicolumn{1}{c}{$\begin{array}{c}\text { Date } \\
\text { Range }\end{array}$} & $\begin{array}{c}\text { Number of } \\
\text { RV Obs. }\end{array}$ & $\begin{array}{c}\text { Reduction } \\
\text { Reference }\end{array}$ \\
\hline HDS & 2009 Aug 8-2010 May 24 & 25 & 1 \\
HIRES & 2010 Feb 24-2010 Jul 3 & 9 & 2 \\
FIES & 2009 Oct 6-2009 Oct 11 & 6 & 3 \\
\hline
\end{tabular}

Notes. ${ }^{\text {a }}$ The mean heliocentric RV of the target. Systematic differences between the velocities from the two instruments are consistent with the velocity zeropoint uncertainties.

References. (1) Sato et al. 2005; (2) Butler et al. 1996; (3) Buchhave et al. 2010.

for the methods used to reduce the data to relative RVs in the solar system barycentric frame. The resulting RV measurements and their uncertainties are listed in Table 4 . The different instrumental uncertainties arise from different slit widths, exposure times, and seeing conditions. The period-folded data, along with our best fit described below in Section 3, are displayed in Figure 3.

One false-alarm possibility is that the observed RVs are not induced by a planetary companion, but are instead caused by distortions in the spectral line profiles due to contamination from a nearby unresolved eclipsing binary. This hypothesis may be tested by examining the spectral line profiles for contamination from a nearby unresolved eclipsing binary (Queloz et al. 2001; Torres et al. 2007). A bisector analysis based on the Keck spectra was performed as described in Section 5 of Bakos et al. (2007). The resulting bisector spans, plotted in Figure 3, show no significant variation, and are not correlated with the RVs, indicating that this is a real TEP system.

In the same figure, one can also see the $S$ index (Vaughan et al. 1978), which is a quantitative measure of the chromospheric activity of the star derived from the flux in the cores of the $\mathrm{Ca}$ II $\mathrm{H}$ and $\mathrm{K}$ lines (Isaacson \& Fischer 2010). Following Noyes et al. (1984) we find that HAT-P-31 has an activity index $\log R_{\mathrm{HK}}^{\prime}=-5.312$, implying that this is a very inactive star.

\section{ANALYSIS}

The analysis of the HAT-P-31 system, including determinations of the properties of the host star and planet, was carried out in a similar fashion to previous HATNet discoveries (e.g., Bakos et al. 2010). Below, we briefly summarize the procedure and the results for the HAT-P-31b system.

\subsection{Properties of the Parent Star}

Stellar atmospheric parameters were measured from our template Keck/HIRES spectrum using the Spectroscopy Made Easy (SME; Valenti \& Piskunov 1996) analysis package and the atomic line database of Valenti \& Fischer (2005). SME yielded the following values and uncertainties: effective temperature $T_{\text {eff } \star}=6065 \pm 100 \mathrm{~K}$, metallicity $[\mathrm{Fe} / \mathrm{H}]=+0.15 \pm 0.08 \mathrm{dex}$, and stellar surface gravity $\log g_{\star}=4.26_{-0.13}^{+0.11}(\mathrm{cgs})$, projected rotational velocity $v \sin i=0.5 \pm 0.6 \mathrm{~km} \mathrm{~s}^{-1}$.

The above atmospheric parameters are then combined with the Yonsei-Yale (YY; Yi et al. 2001) series of stellar evolution models to determine other parameters such as the stellar mass, radius, and age. The results are listed in Table 5. We find that the star has a mass and radius of $M_{\star}=1.218_{-0.063}^{+0.089} M_{\odot}$ and $R_{\star}=1.36_{-0.18}^{+0.27} R_{\odot}$, and an estimated age of $3.17_{-1.11}^{+0.70} \mathrm{Gyr}$.
Table 4

Relative Radial Velocities, Bisector Spans, and Activity Index Measurements of HAT-P-31

\begin{tabular}{|c|c|c|c|c|c|c|}
\hline $\begin{array}{l}\mathrm{BJD}^{\mathrm{a}} \\
{[2,454,000+]}\end{array}$ & $\begin{array}{c}\mathrm{RV}^{\mathrm{b}} \\
\left(\mathrm{m} \mathrm{s}^{-1}\right)\end{array}$ & $\begin{array}{c}\sigma_{\mathrm{RV}}^{\mathrm{c}} \\
\left(\mathrm{m} \mathrm{s}^{-1}\right)\end{array}$ & $\begin{array}{c}\mathrm{BS} \\
\left(\mathrm{m} \mathrm{s}^{-1}\right)\end{array}$ & $\begin{array}{c}\sigma_{\mathrm{BS}} \\
\left(\mathrm{m} \mathrm{s}^{-1}\right)\end{array}$ & $S^{\mathrm{d}}$ & Instrument \\
\hline 1051.87826 & -30.72 & 6.99 & $\ldots$ & $\ldots$ & $\ldots$ & Subaru \\
\hline 1051.88946 & -27.97 & 6.90 & $\ldots$ & . & $\ldots$ & Subaru \\
\hline 1051.90067 & -44.27 & 6.56 & $\ldots$ & $\ldots$ & $\ldots$ & Subaru \\
\hline 1052.81471 & -163.76 & 9.25 & $\ldots$ & $\ldots$ & $\ldots$ & Subaru \\
\hline 1052.93238 & -200.89 & 6.63 & $\ldots$ & $\ldots$ & $\ldots$ & Subaru \\
\hline 1052.94706 & -202.52 & 7.29 & $\ldots$ & $\ldots$ & $\ldots$ & Subaru \\
\hline 1053.80433 & -159.83 & 7.09 & $\ldots$ & $\ldots$ & $\ldots$ & Subaru \\
\hline 1053.81555 & -166.62 & 7.47 & $\ldots$ & $\ldots$ & $\ldots$ & Subaru \\
\hline 1053.82676 & -163.36 & 7.95 & $\ldots$ & $\ldots$ & $\ldots$ & Subaru \\
\hline 1053.89544 & -145.48 & 7.08 & $\ldots$ & $\ldots$ & $\ldots$ & Subaru \\
\hline 1053.90665 & -140.21 & 6.46 & $\ldots$ & $\ldots$ & $\ldots$ & Subaru \\
\hline 1053.91786 & -136.05 & 6.39 & $\ldots$ & $\ldots$ & $\ldots$ & Subaru \\
\hline 1111.33488 & 71.48 & 9.40 & $\ldots$ & $\ldots$ & $\ldots$ & FIES \\
\hline 1112.33770 & -115.00 & 10.70 & $\ldots$ & $\ldots$ & $\ldots$ & FIES \\
\hline 1113.34016 & -225.79 & 10.50 & $\ldots$ & $\ldots$ & $\ldots$ & FIES \\
\hline 1114.34798 & 12.92 & 10.70 & $\ldots$ & $\ldots$ & $\ldots$ & FIES \\
\hline 1115.38654 & 234.94 & 10.80 & $\ldots$ & $\ldots$ & $\ldots$ & FIES \\
\hline 1116.33401 & 101.33 & 24.70 & $\ldots$ & $\ldots$ & $\ldots$ & FIES \\
\hline 1252.10465 & $\ldots$ & $\ldots$ & -2.81 & 1.78 & 0.1280 & Keck \\
\hline 1252.11375 & -34.70 & 2.65 & 5.07 & 1.72 & 0.1190 & Keck \\
\hline 1286.09877 & 153.49 & 2.56 & 2.47 & 1.48 & 0.1270 & Keck \\
\hline 1338.90673 & -199.81 & 11.70 & $\ldots$ & $\ldots$ & $\ldots$ & Subaru \\
\hline 1338.91100 & -199.43 & 11.83 & $\ldots$ & $\ldots$ & $\ldots$ & Subaru \\
\hline 1338.91526 & -196.43 & 10.73 & $\ldots$ & $\ldots$ & $\ldots$ & Subaru \\
\hline 1338.91953 & -188.53 & 11.15 & $\ldots$ & $\ldots$ & $\ldots$ & Subaru \\
\hline 1339.85945 & 129.95 & 16.85 & $\ldots$ & $\ldots$ & $\ldots$ & Subaru \\
\hline 1339.87200 & 140.14 & 11.90 & $\ldots$ & $\ldots$ & $\ldots$ & Subaru \\
\hline 1339.88669 & 151.20 & 9.63 & $\ldots$ & $\ldots$ & $\ldots$ & Subaru \\
\hline 1339.95891 & 180.56 & 7.45 & $\ldots$ & $\ldots$ & $\ldots$ & Subaru \\
\hline 1339.97360 & 179.56 & 7.50 & $\ldots$ & $\ldots$ & $\ldots$ & Subaru \\
\hline 1339.98489 & 186.81 & 8.74 & $\ldots$ & $\ldots$ & $\ldots$ & Subaru \\
\hline 1341.08414 & 167.01 & 13.93 & $\ldots$ & $\ldots$ & $\ldots$ & Subaru \\
\hline 1341.09189 & 158.61 & 13.28 & $\ldots$ & $\ldots$ & $\ldots$ & Subaru \\
\hline 1341.10303 & 151.27 & 9.77 & $\ldots$ & $\ldots$ & $\ldots$ & Subaru \\
\hline 1343.00801 & -167.85 & 2.30 & 10.12 & 1.17 & 0.1350 & Keck \\
\hline 1372.86477 & -139.73 & 2.11 & -3.69 & 1.30 & 0.1230 & Keck \\
\hline 1374.99015 & 177.54 & 1.84 & 0.74 & 1.16 & 0.1240 & Keck \\
\hline 1375.86758 & 207.17 & 1.90 & -3.49 & 1.11 & 0.1240 & Keck \\
\hline 1378.79885 & -228.26 & 1.90 & -4.23 & 1.29 & 0.1230 & Keck \\
\hline 1380.79961 & 212.57 & 1.93 & -4.20 & 1.12 & 0.1220 & Keck \\
\hline
\end{tabular}

Notes. For the iodine-free template exposures there is no RV measurement, but the BS and $S$ index can still be determined.

${ }^{a}$ Barycentric Julian dates throughout the paper are calculated from Coordinated Universal Time.

b The zero point of these velocities is arbitrary. An overall offset $\gamma_{\text {rel }}$ fitted to these velocities in Section 3.2 has not been subtracted.

${ }^{c}$ Internal errors excluding the component of astrophysical/instrumental jitter considered in Section 3.2.

${ }^{\mathrm{d}}$ Relative chromospheric activity index, not calibrated to the scale of Vaughan et al. (1978).

For previous HATNet planets (e.g., Bakos et al. 2010) we used the normalized semimajor axis, $a / R_{\star}$ which is closely related to $\rho_{\star}$, the mean stellar density, and is determined from the analysis of the light curves and RV curves, to obtain an improved estimate of $\log g_{\star}$, which is then held fixed in a second SME iteration. In this case, because we only have the relatively low-precision HATNet light curve, $a / R_{\star}$ is poorly constrained, and we instead opt to use the SME determination of $\log g_{\star}$ rather than $a / R_{\star}$ as a luminosity indicator. Figure 4 shows the location of the star in a diagram of $\log g_{\star}$ versus $T_{\text {eff } \star}$, together with the model 


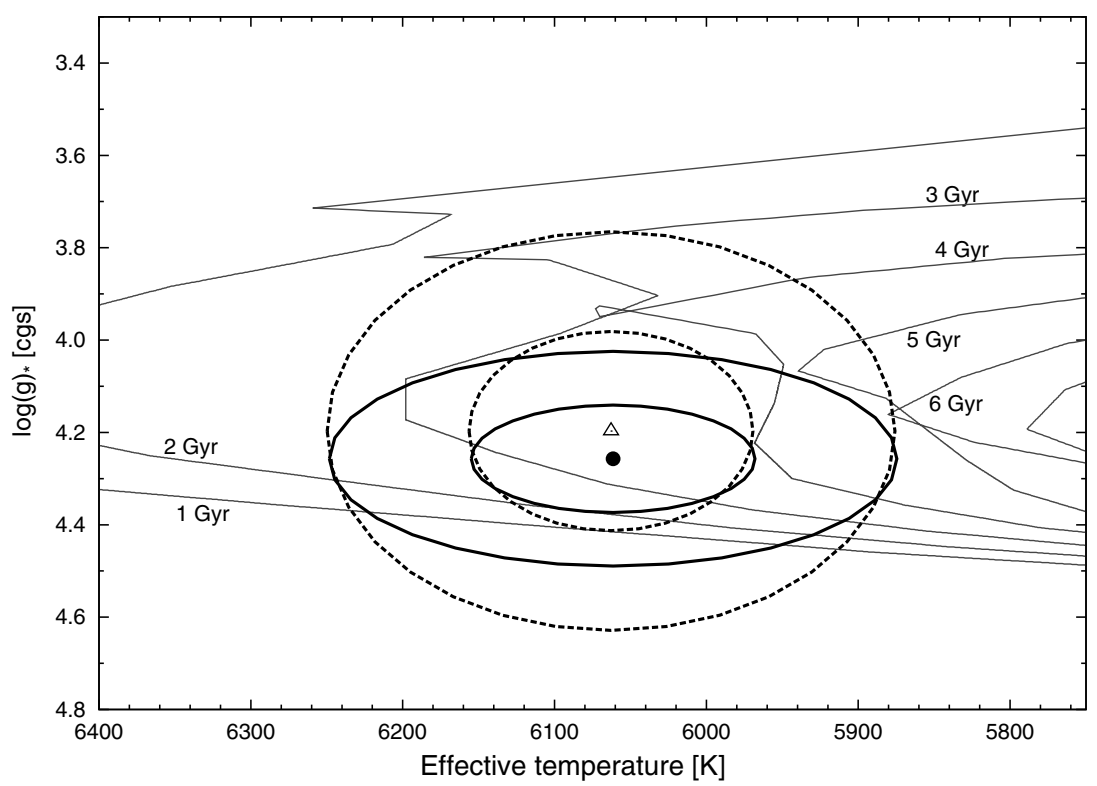

Figure 4. Model isochrones from Yi et al. (2001) for the measured metallicity of HAT-P-31, $[\mathrm{Fe} / \mathrm{H}]=0.15$, and ages from 1 to $14 \mathrm{Gyr}$ in steps of $1 \mathrm{Gyr}$ (gray-scale lines, left to right). The adopted values of $T_{\text {eff }}$ and $\log g_{\star}$ determined from the SME analysis are shown together with their $1 \sigma$ and $2 \sigma$ confidence ellipsoids (filled circle, and bold solid lines). The less-tight $1 \sigma$ and $2 \sigma$ constraints imposed by $a / R_{\star}$ are also shown (open triangle, and bold dotted lines).

Table 5

Stellar Parameters for HAT-P-31

\begin{tabular}{|c|c|c|}
\hline Parameter & Value & Source \\
\hline \multicolumn{3}{|c|}{ Spectroscopic properties } \\
\hline$T_{\mathrm{eff} \star}(\mathrm{K}) \ldots \ldots$ & $6065 \pm 100$ & $\mathrm{SME}^{\mathrm{a}}$ \\
\hline$[\mathrm{Fe} / \mathrm{H}] \ldots \ldots \ldots$ & $0.15 \pm 0.08$ & SME \\
\hline$v \sin i\left(\mathrm{~km} \mathrm{~s}^{-1}\right) .$. & $0.5 \pm 0.6$ & SME \\
\hline$v_{\text {mac }}\left(\mathrm{km} \mathrm{s}^{-1}\right)^{\mathrm{b}} \ldots$ & 4.47 & SME \\
\hline$v_{\text {mic }}\left(\mathrm{km} \mathrm{s}^{-1}\right)^{\mathrm{b}}$ & 0.85 & SME \\
\hline$\gamma_{\mathrm{RV}}\left(\mathrm{km} \mathrm{s}^{-1}\right) \ldots$ & $-2.40 \pm 0.03$ & TRES \\
\hline \multicolumn{3}{|c|}{ Photometric properties } \\
\hline$V(\operatorname{mag}) \ldots \ldots$ & 11.660 & TASS \\
\hline$V-I_{C}(\mathrm{mag}) \ldots$ & $0.67 \pm 0.17$ & TASS \\
\hline$J(\mathrm{mag}) \ldots \ldots$ & $10.423 \pm 0.023$ & 2MASS \\
\hline$H(\mathrm{mag}) \ldots \ldots$ & $10.128 \pm 0.027$ & 2MASS \\
\hline$K_{s}(\mathrm{mag}) \ldots \ldots$ & $10.083 \pm 0.021$ & 2MASS \\
\hline \multicolumn{3}{|c|}{ Derived properties } \\
\hline$M_{\star}\left(M_{\odot}\right) \ldots \ldots$ & $1.218_{-0.063}^{+0.089}$ & $\mathrm{YY}+\mathrm{SME}^{\mathrm{c}}$ \\
\hline$R_{\star}\left(R_{\odot}\right)$ & $1.36_{-0.18}^{+0.27}$ & $\mathrm{YY}+\mathrm{SME}$ \\
\hline $\log \left(g_{*}(\operatorname{cgs})\right) \ldots$ & $4.26_{-0.13}^{+0.11}$ & $\mathrm{YY}+\mathrm{SME}$ \\
\hline$L_{\star}\left(L_{\odot}\right) \ldots \ldots$ & $2.23_{-0.58}^{+1.01}$ & $\mathrm{YY}+\mathrm{SME}$ \\
\hline$M_{V}(\mathrm{mag}) \ldots \ldots$ & $3.91_{-0.41}^{+0.34}$ & YY+SME \\
\hline$M_{K}(\mathrm{mag}, \mathrm{ESO})$. & $2.64 \pm 0.30$ & $\mathrm{YY}+\mathrm{SME}$ \\
\hline Age $(\mathrm{Gyr}) \ldots .$. & $3.17_{-1.11}^{+0.70}$ & $\mathrm{YY}+\mathrm{SME}$ \\
\hline Distance $(\mathrm{pc}) \ldots$ & $354_{-51}^{+74}$ & $\mathrm{YY}+\mathrm{SME}$ \\
\hline
\end{tabular}

Notes.

a SME: "Spectroscopy Made Easy" package for the analysis of high-resolution spectra (Valenti \& Piskunov 1996).

${ }^{\mathrm{b}}$ Assumed quantity based upon derived spectral type.

${ }^{c}$ YY+SME: based on the YY isochrones (Yi et al. 2001) and the SME results.

isochrones. For comparison we also show the relatively poor constraint on $\log g_{\star}$ that is imposed by $a / R_{\star}$.
As an additional check on the stellar evolution modeling, we note that HAT-P-31 has a measured near-infrared color of $J-K=0.364 \pm 0.034$, which we have taken from 2MASS (Skrutskie et al. 2006) using the Carpenter (2001) transformation to the ESO photometric system. This is within $2 \sigma$ of the predicted value from the isochrones of $J-K=$ $0.31 \pm 0.11$. The distance listed in Table 5 is calculated by comparing the observed $K$ magnitude (taken from 2MASS and transformed to ESO) to the absolute $K$ magnitude from the stellar models.

\subsection{Global Modeling of the Data}

\subsubsection{Photometry}

In previous HATNet papers, we have used a simplified model for the transit light curve of the HATNet data. For HAT-P-31, no precise photometry exists and thus we fit the HATNet data using a more sophisticated quadratic limb-darkening Mandel \& Agol (2002) algorithm with limb-darkening coefficients interpolated from the tables by Claret (2004). One caveat is that the instrumental blending factor, $B_{\text {inst }}$, is unknown as discussed earlier in Section 2. We point out that experience with previous HATNet planets suggests $B_{\text {inst }}$ is within $2 \sigma$ of unity for light curves processed using reconstructive TFA in all cases and thus can be accounted for by conservatively doubling the uncertainties on $p$ and $R_{P}$. Further support for a $B_{\text {inst }}$ factor not greatly different from unity come from the fact HAT-P-31 is fairly isolated and there are no neighbors in 2MASS or a DSS image which contribute significant flux to the HATNet aperture.

Due to the low-precision photometry, the stellar density cannot be determined to high precision using the method of Seager \& Mallén-Ornelas (2003) and in fact spectroscopic estimates were found to be more precise. However, we can reverse this well-known trick by implementing a Bayesian prior in our fitting process for the stellar density.

We use the spectroscopically determined stellar density from Section 3.1 as a prior in our fits. Since the period of the transiting planet is well constrained for even low signal-tonoise photometry, reasonably precise estimates for $P_{b}$ and $\rho_{*}$ are 

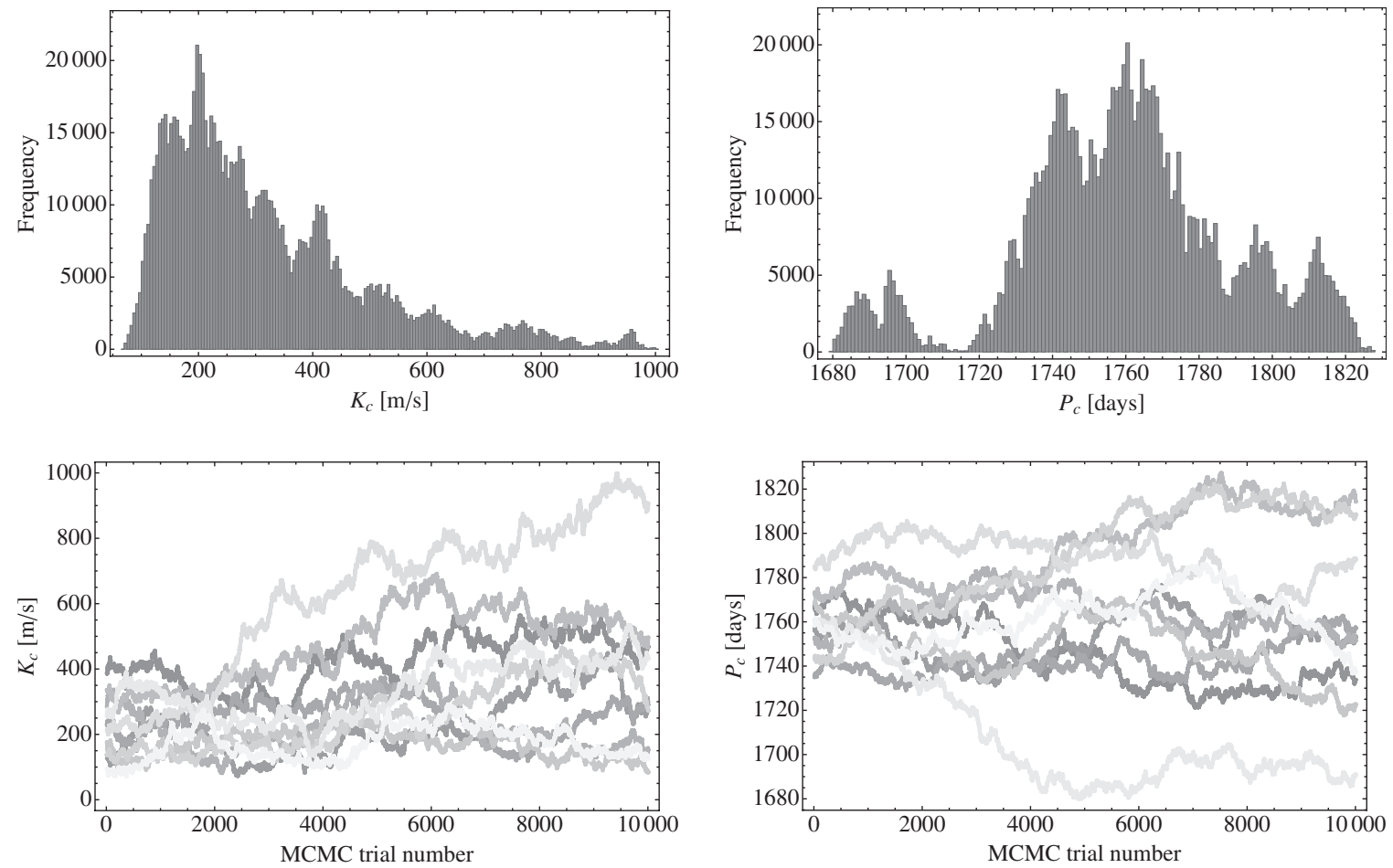

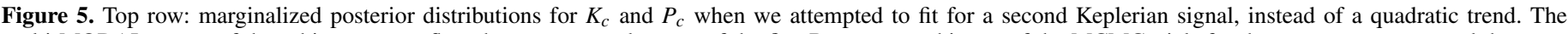

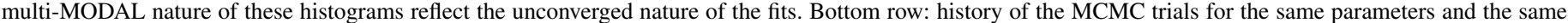

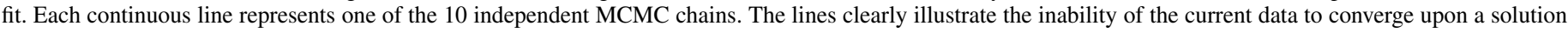
for HAT-P-31c.

Table 6

Comparison of RV Models Attempted for HAT-P-31

\begin{tabular}{|c|c|c|}
\hline Model & $\chi^{2}$ & $\mathrm{BIC}^{\mathrm{a}}$ \\
\hline Circular planet . . . . . . . . . . . & 3714.9 & 3729.6 \\
\hline Eccentric planet $\ldots . \ldots \ldots \ldots \ldots$ & 204.2 & 226.3 \\
\hline Eccentric planet + drift. . . . . . . . & 159.6 & 185.5 \\
\hline Eccentric planet + trojan. . . . . . . & 191.1 & 216.9 \\
\hline Eccentric planet + drift + trojan $\ldots$ & 113.2 & 142.7 \\
\hline Eccentric planet + drift + quadratic & 33.9 & 63.4 \\
\hline Eccentric planet + circular planet. . & 33.6 & 66.8 \\
\hline Eccentric planet + eccentric planet & 33.2 & 73.8 \\
\hline
\end{tabular}

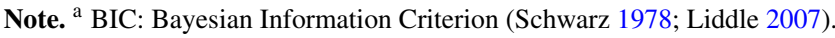

possible. With these two constrained, $\left(a_{b} / R_{*}\right)$ is therefore also constrained. The transit light curve is essentially characterized by four parameters, $\tau_{b}, \delta_{b}, b_{b}$, and $\left(a_{b} / R_{*}\right)$ and thus one of these parameters is constrained by the combination of the transit times and the stellar density prior alone.

The photometry which is fitted in the global modeling is corrected for instrumental systematics through the EPD and reconstructive TFA correction procedures prior to performing the fit (see Section 2.1 and Bakos et al. 2010 for details).

\subsubsection{Radial Velocities}

For the RV fits, we found a single planet fit gave a very poor fit to the observations ( $\chi^{2}=204.2$ for $40 \mathrm{RV}$ points) and quickly appreciated some kind of second signal was present. Exploring different models, such as Trojan offsets, polynomial time trends, and outer companions, (see Table 6 for comparison), we found that an eccentric transiting planet with a quadratic trend in the RVs was the preferred model. The pivot point $\left(t_{\text {pivot }}\right)$ of the polynomial models, including the drift and quadratic trends, was selected to be the weighted mean of the RV time stamps.

The most likely physical explanation for a quadratic trend is a third body in the system, described by a Keplerian model. Indeed, the Keplerian model provides an improved $\chi^{2}$ for a circular orbit and then again for an eccentric orbit but the extra degrees of freedom penalize our model selection criterion. We also found that these models were highly unconstrained and convergence in the associated fits was unsatisfactory. An illustration of the lack of convergence is shown in Figure 5. Therefore, we will adopt the quadratic model in our final reported parameters in Table 7.

The quadratic model may be used to infer some physical parameters of the third planet. To make some meaningful progress, we will assume the outer planet is on a circular orbit. One may compare the quadratic and Keplerian model descriptions via

$$
\begin{gathered}
\mathrm{RV}_{\text {quad }}^{c}=\gamma^{\prime}+\dot{\gamma}\left(t-t_{\text {pivot }}\right)+0.5 \ddot{\gamma}\left(t-t_{\text {pivot }}\right)^{2} \\
\mathrm{RV}_{\text {Kep }}^{c}=\gamma^{\prime}-K_{c} \sin \left(\frac{2 \pi\left(t-\tau_{c}\right)}{P_{c}}\right) .
\end{gathered}
$$

By differentiating both expressions and solving for the time when the signals are minimized, one may write

$$
\tau_{c}+\frac{P_{c}}{4}=\frac{-\dot{\gamma}+\ddot{\gamma} t_{\text {pivot }}}{\ddot{\gamma}} .
$$


Table 7

Global Fit Results for HAT-P-31

\begin{tabular}{|c|c|}
\hline Parameter $^{\mathrm{a}}$ & Value \\
\hline \multicolumn{2}{|c|}{ Fitted parameters } \\
\hline$P_{b}$ (days) & $5.005425_{-0.000092}^{+0.000091}$ \\
\hline$\tau_{b}\left(\mathrm{BJD}_{\mathrm{TDB}}-2,450,000\right)$ & $4320.8866_{-0.0053}^{+0.0051}$ \\
\hline$\left[\tilde{T}_{1}\right]_{b}(\mathrm{~s})$ & $16300_{-1000}^{+1000}$ \\
\hline$p_{b}^{2}(\%)$ & $0.65_{-0.12}^{+0.18}$ \\
\hline$b_{b}$ & $0.57_{-0.31}^{+0.23}$ \\
\hline OOT & $1.00061_{-0.00016}^{+0.00016}$ \\
\hline$K_{b}\left(\mathrm{~ms}^{-1}\right)$ & $232.5_{-1.1}^{+1.1}$ \\
\hline$e_{b} \sin \omega_{b}$ & $-0.2442_{-0.0043}^{+0.0043}$ \\
\hline$e_{b} \cos \omega_{b}$ & $0.0185_{-0.0079}^{+0.0080}$ \\
\hline$\gamma_{\text {Keck }}\left(\mathrm{ms}^{-1}\right)$ & $-29.0_{-1.4}^{+1.4}$ \\
\hline$\gamma_{\text {Subaru }}\left(\mathrm{ms}^{-1}\right)$ & $18.8_{-3.1}^{+3.1}$ \\
\hline$\gamma_{\text {FIES }}\left(\mathrm{ms}^{-1}\right)$ & $92.7_{-5.6}^{+5.6}$ \\
\hline$\dot{\gamma}\left(\mathrm{ms}^{-1} \mathrm{day}^{-1}\right)$ & $0.141_{-0.025}^{+0.025}$ \\
\hline$\ddot{\gamma}\left(\mathrm{ms}^{-1} \mathrm{day}^{-2}\right)$ & $0.00226_{-0.00021}^{+0.00021}$ \\
\hline \multicolumn{2}{|c|}{ SME derived quantities } \\
\hline$u_{1}^{\mathrm{b}, \mathrm{e}}$ & $0.2078^{*}$ \\
\hline$u_{2}^{\mathrm{b}, \mathrm{e}}$ & $0.3550^{*}$ \\
\hline$\rho_{*}^{\mathrm{c}, \mathrm{e}}\left(\mathrm{g} \mathrm{cm}^{-3}\right)$ & $0.69_{-0.26}^{+0.34}$ \\
\hline \multicolumn{2}{|c|}{ HAT-P-31b derived properties } \\
\hline$\Psi_{b}$ & $0.4737_{-0.0064}^{+0.0065}$ \\
\hline$e_{b}$ & $0.2450_{-0.0045}^{+0.0045}$ \\
\hline$\omega_{b}\left({ }^{\circ}\right)$ & $274.3_{-1.8}^{+1.8}$ \\
\hline $\log \left(g_{b}(\operatorname{cgs})\right)$ & $3.61_{-0.32}^{+0.15}$ \\
\hline$\left(a_{b} / R_{*}\right)$ & $8.9_{-2.3}^{+1.4}$ \\
\hline$i_{b}\left(^{\circ}\right)$ & $87.1_{-2.7}^{+1.8}$ \\
\hline$\left[T_{1,4}\right]_{b}(\mathrm{~s})$ & $18500_{-1200}^{+1700}$ \\
\hline$\left[T_{2,3}\right]_{b}(\mathrm{~s})$ & $14200_{-2400}^{+1300}$ \\
\hline$p_{b}$ & $0.080_{-0.015}^{+0.022}$ \\
\hline$M_{b}\left(M_{J}\right)$ & $2.171_{-0.077}^{+0.105}$ \\
\hline$R_{b}\left(R_{J}\right)$ & $1.07_{-0.16}^{+0.24}$ \\
\hline $\operatorname{Corr}\left(R_{b}, M_{b}\right)$ & 0.795 \\
\hline$\rho_{b}\left(\mathrm{~g} \mathrm{~cm}^{-3}\right)$ & $2.18_{-0.93}^{+1.24}$ \\
\hline$a_{b}(\mathrm{AU})$ & $0.055_{-0.015}^{+0.015}$ \\
\hline$\left[T_{\mathrm{eq}}\right]_{b}(\mathrm{~K})$ & $1450_{-110}^{+230}$ \\
\hline$\Theta^{\mathrm{e}}$ & $0.190_{-0.056}^{+0.036}$ \\
\hline$F_{\text {peri }}\left(10^{9} \mathrm{erg} \mathrm{s}^{-1} \mathrm{~cm}^{-2}\right)$ & $1.69_{-0.44}^{+1.39}$ \\
\hline$F_{\text {ap }}\left(10^{9} \mathrm{erg} \mathrm{s}^{-1} \mathrm{~cm}^{-2}\right)$ & $0.62_{-0.16}^{+0.51}$ \\
\hline$\langle F\rangle^{\mathrm{f}}\left(10^{9} \mathrm{erg} \mathrm{s}^{-1} \mathrm{~cm}^{-2}\right)$ & $0.99_{-0.26}^{+0.82}$ \\
\hline \multicolumn{2}{|c|}{ HAT-P-31c derived quantities } \\
\hline$\tau_{c}+0.25 P_{c}\left(\mathrm{BJD}_{\mathrm{TDB}}-2,450,000\right)$ & $5254.9_{-6.8}^{+7.4}$ \\
\hline$K_{c} P_{c}^{-2}\left(\mathrm{~ms}^{-1} \mathrm{day}^{-2}\right)$ & $7.64_{-0.70}^{+0.71}$ \\
\hline$P_{c}($ years $)$ & $\geqslant 2.8$ \\
\hline$K_{c}\left(\mathrm{~ms}^{-1}\right)$ & $\geqslant 60$ \\
\hline$M_{c}\left(M_{J}\right)$ & $\geqslant 3.4$ \\
\hline
\end{tabular}

Notes.

${ }^{a}$ Quoted values are medians of MCMC trials with errors given by $1 \sigma$ quantiles.

"b" subscripts refer to planet HAT-P-31b and "c" subscripts refer to HAT-P-31c.

${ }^{\mathrm{b}}$ Fixed parameter.

c Parameter is treated as a prior.

${ }^{\mathrm{d}}$ Parameter determined using SME and YY isochrones.

e The Safronov number is given by $\Theta=\frac{1}{2}\left(V_{\text {esc }} / V_{\text {orb }}\right)^{2}=\left(a / R_{p}\right)\left(M_{p} / M_{\star}\right)$ (see Hansen \& Barman 2007).

${ }^{\mathrm{f}}$ Incoming flux per unit surface area, averaged over the orbit.
Differentiating both RV models with respect to time twice and evaluating at the moment when both signals are minimized yields

$$
\frac{K_{c}}{P_{c}^{2}}=\frac{\ddot{\gamma}}{4 \pi^{2}} .
$$

Equations (3) and (4) may therefore be used to determine some information about HAT-P-31c.

To evaluate the statistical significance of HAT-P-31c, we performed an $F$-test between the one-planet and two-planet models. In both cases, HAT-P-31b is assumed to maintain non-zero orbital eccentricity. Assuming HAT-P-31c is on a circular orbit, the false-alarm probability (FAP) from an $F$-test is $3.0 \times 10^{-12}$, or $7.0 \sigma$. Assuming HAT-P-31c is on an eccentric orbit requires two more degrees of freedom and thus reduces the FAP to $1.3 \times 10^{-10}$, or $6.4 \sigma$. From a statistical perspective then, the presence of HAT-P-31c is highly secure. We point out this determination of course assumes purely Gaussian uncertainties and no outlier measurements.

\subsubsection{Fitting Algorithm}

We utilize a Metropolis-Hastings Markov Chain Monte Carlo (MCMC) algorithm to globally fit the data, including the stellar density prior (our routine is described in Kipping \& Bakos (2011)). To ensure the parameter space is fully explored, we used five independent MCMC fits which stop once $1.25 \times 10^{5}$ trials have been accepted and burn out the first $20 \%$. This leaves us with a total of $5 \times 10^{5}$ points for the posterior distributions. At the end of the fit, a more aggressive downhill simplex $\chi^{2}$ minimization is used, for which the final solution is used for Figures 2 and 3.

There were 14 free parameters in the global fit: $\left\{\tau_{b}, p_{b}^{2}\right.$, $\left[\tilde{T}_{1}\right]_{b}, b_{b}, P_{b}, e_{b} \sin \omega_{b}, e_{b} \cos \omega_{b}, \gamma_{\text {Keck }}, \gamma_{\text {FIES }}, \gamma_{\text {Subaru }}, K_{b}, \dot{\gamma}$, $\ddot{\gamma}$, OOT $\}$, which we elaborate on here. $\tau_{b}$ is the time of transit minimum (Kipping 2011), frequently dubbed by the misnomer "mid-transit time." $p_{b}^{2}$ is the ratio-of-radii squared and $P_{b}$ is the orbital period. [ $\left.\tilde{T}_{1}\right]_{b}$ is the "one-term" approximate equation (Kipping 2010) for the transit duration between the instant when the center of the planet crosses the stellar limb to exiting under the same condition. $b_{b}$ is the impact parameter, defined as the sky-projected planet-star separation in units of the stellar radius at the instant of inferior conjunction. $e_{b}$ is the orbital eccentricity and $\omega_{b}$ is the associated position of pericenter. $\gamma$ terms relate to the instrumental offsets for the RVs. Similarly, OOT is the out-of-transit flux for the HATNet photometry. Finally, $K_{b}$ is the RV semi-amplitude, and $\dot{\gamma}$ (drift) and $\ddot{\gamma}$ (curl) are the first and second time derivatives of $\gamma$.

Final quoted results are the median of the marginalized posterior for each fitted parameter with $34.15 \%$ quantiles either side for the $1 \sigma$ uncertainties (see Table 7). The uncertainties on $p, p^{2}$, and $R_{P}$ have been conservatively doubled for reasons described in Section 2. Histograms of the posterior distributions for the fitted parameters are provided in Figure 6. We find that the stellar jitter of this star is at or below the measurement uncertainties $\left(\sim 2 \mathrm{~m} \mathrm{~s}^{-1}\right)$.

Table 7 provides estimates for some minimum limits on various parameters of interest relating to HAT-P-31c. These limits are determined by the known constraints on the minimum $P_{c}$. We determined this value by forcing a circular orbit Keplerian fit for planet $\mathrm{c}$ through the data, stepping through a range of periods from 1 year to 5 years in 1 day steps. The 

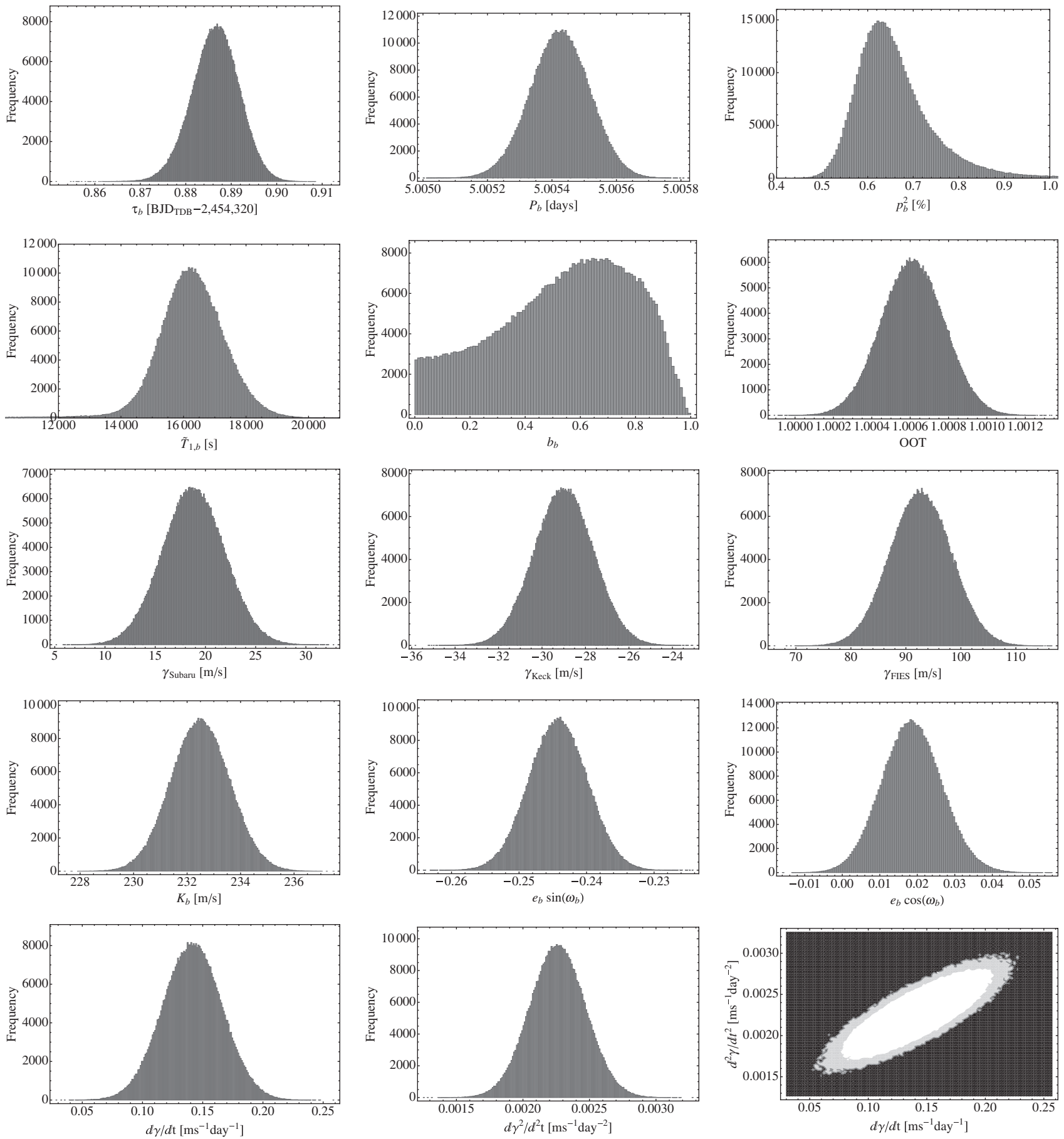

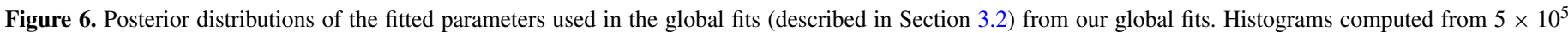
MCMC trials. Bottom-right panel shows joint-posterior of the radial velocity drift and curl, with white denoting the $2 \sigma$ region of confidence and gray the $3 \sigma$.

minimum limit on $P_{c}$ is defined as when $\Delta \chi^{2}=1$, relative to the quadratic trend fit, occurring at 2.8 years.

\section{DISCUSSION}

\subsection{Physical Properties of HAT-P-31b and $c$}

HAT-P-31b is a $2.17 M_{J}$ hot Jupiter transiting the host star once every 5.005 days. Due to the lack of follow-up photometry obtained for this object (as a consequence of the nearly integer orbital period), we have only HATNet photometry, which is of lower $\mathrm{S} / \mathrm{N}$ than dedicated follow-up. This fact, combined with our choice to double all uncertainties on depth related transit terms, leads to a large uncertainty on the planetary radius of $R_{b}=1.07_{-0.32}^{+0.48} R_{J}$, consistent with many other hot-Jupiter objects (see http://exoplanet.eu).

High-precision RVs also indicate the presence of an outer body, HAT-P-31c, found through an induced quadratic trend in the RV residuals. Keplerian fits are unable to convincingly distinguish between a circular or eccentric orbit for this object. HAT-P-31c has a minimum mass of $M_{c} \geqslant 3.4 M_{J}$ and eccentric orbit solutions significantly increase this figure. It is unclear whether HAT-P-31c is a brown dwarf or a "planet," and future work will be needed to determine this. 

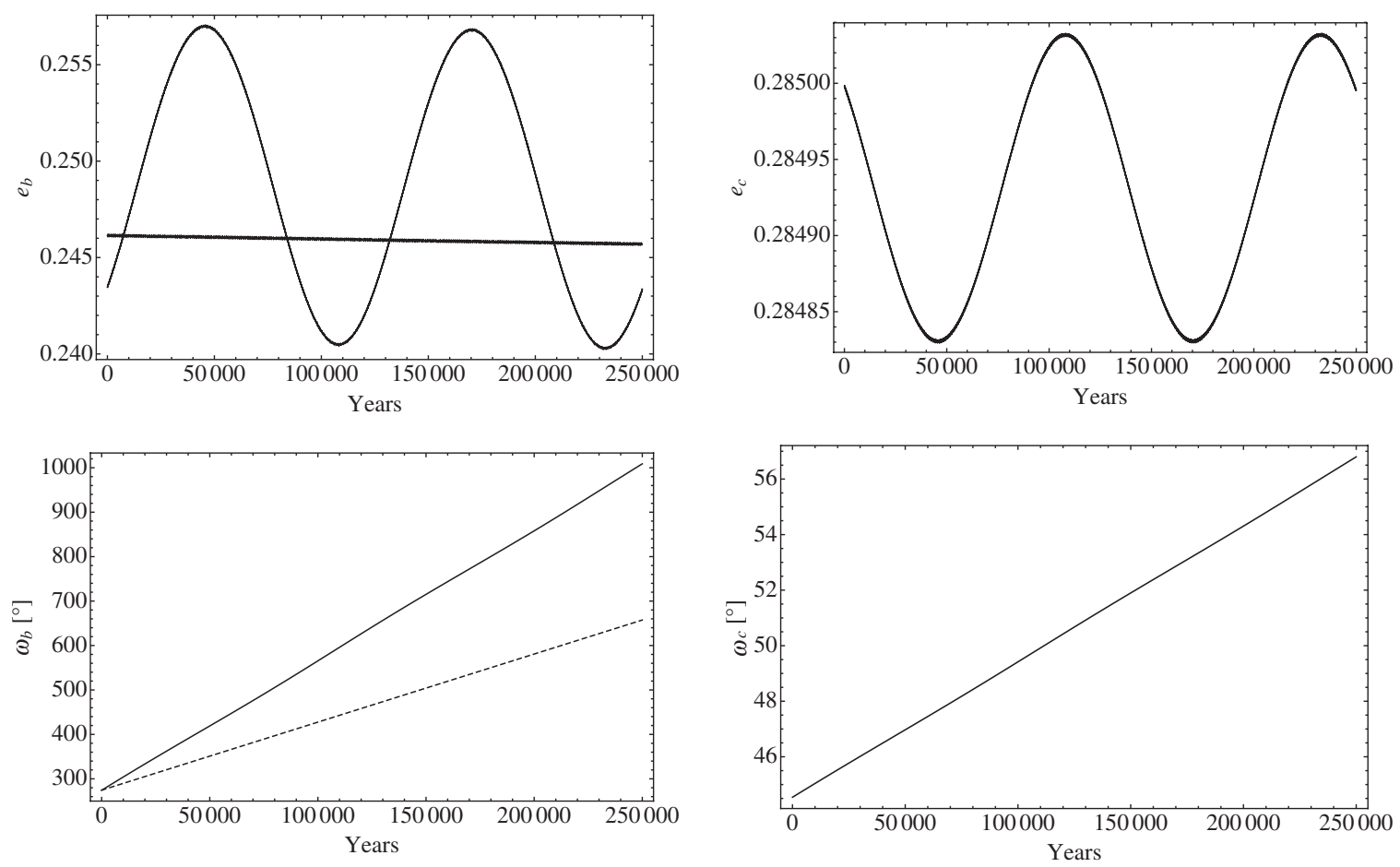

Figure 7. Two possible realizations for the orbital evolution of planets HAT-P-31b and c. The solid lines show the evolution starting from an eccentric orbit solution for HAT-P-31c. The dashed lines show the evolution starting from a circular orbit solution for HAT-P-31c.

\subsection{Orbital Stability}

\subsubsection{Circular Fit for HAT-P-31c}

Here we discuss our procedure to test the dynamical stability of two possible orbital configurations. It should be noted that the eccentricity of HAT-P-31b is highly secure but the eccentricity of HAT-P-31c remains unclear. For this reason, we repeat our simulations assuming both a circular and eccentric orbit for HAT-P-31c, beginning with the former. We utilize the Systemic Console (Meschiari et al. 2009) for this purpose assuming a coplanar configuration. Employing the Gragg-Bulirsch-Stoer integrator, orbital evolution was computed for 250,000 years for the HAT-P-31 system (see Figure 7).

We first consider the circular case. The orbital period and mass of HAT-P-31c are non-convergent parameters and so we can only provide an orbital solution which gives a good fit to the data, but is not necessarily unique. To this end, we proceeded to input HAT-P-31c with $P_{c}=4.86$ years and $M_{c}=13.0 M_{J}$, corresponding to the solution presented in Table 6 . This test revealed minor evolution over the course of our simulations, indicating a stable and essentially static configuration.

\subsubsection{Eccentric Fit for HAT-P-31c}

To test the eccentric fit, we again used the lowest $\chi^{2}$ solution presented earlier in Table 6 , corresponding to $P_{c}=4.82$ years and $e_{c}=0.285$. We found that the system was also stable over 250,000 years (see Figure 7). However, the simulations do show the eccentricity of planet $b$ varying sinusoidally over a timescale of $\sim 125,000$ years with an amplitude of $\sim 0.01$. The eccentricity of planet $\mathrm{c}$ also varies in anti-phase but with a much smaller amplitude. The eccentric evolution shows faster apsidal precession for planet $b$, but this is unlikely to be observable through changes in the transit duration. We estimate the duration will change by $0.2 \mathrm{~s}$ over 10 years (corresponding to $\Delta \omega_{b}=0.027$ ) using the expressions of Kipping (2010).
The orbital period and semimajor axes of both bodies were stable over the 250,000 years of integration considered here.

\subsubsection{Habitable-zone Bodies}

We tried adding a habitable-zone Earth-mass planet on a circular orbit into the system and testing stability. One may argue that the probable history of this system involved the inward migration of HAT-P-31b and that this migration through the inner protoplanetary disk would essentially eliminate the possibility of an Earth-like planet forming in the habitablezone. However, Fogg \& Nelson (2007) have shown that this not necessarily true. In their simulations, it is found that $>60 \%$ of the solid disk survives, including planetesimals and protoplanets, by being scattered by the giant planet into external orbits where dynamical friction is strong and terrestrial planet formation is able to resume. In one simulation, a planet of $2 M_{\oplus}$ formed in the habitable-zone after a hot-Jupiter passed through and its orbit stabilized at $0.1 \mathrm{AU}$.

For a planet to receive the same insolation as the Earth, we estimate $P=604$ days. For our circular orbit solution of HATP-31c, the habitable-zone Earth-mass planet is stable for over 100,000 years. For our eccentric orbit solution, the Earth-like planet is summarily ejected in less than 1000 years.

\subsection{Circularization Timescale}

Due to the poor constraints on the planetary radius, there is a great deal of uncertainty in the circularization timescale $\left(\tau_{\text {circ }}\right)$ for HAT-P-31b. Nevertheless, using the equations of Adams \& Laughlin (2006), we used the MCMC results to compute the posterior distribution of $\tau_{\text {circ }}$. We find that the age of HAT-P-31 is equal to $24_{-15}^{+122}$ circularization timescales, assuming $Q_{P}=10^{5}$. This indicates that we currently have insufficient data to assess whether the observed eccentricity is anomalous or not. Improved radius constraints will certainly 
aid in this calculation and may lend or detract credence to the hypothesis of eccentricity pumping of the inner planet by HAT-P-31c.

HATNet operations have been funded by NASA grants NNG04GN74G, NNX08AF23G, and SAO IR\&D grants. D.M.K. has been supported by Smithsonian Institution Restricted Endowment Funds. Work of G.Á.B. and J. Johnson were supported by the Postdoctoral Fellowship of the NSF Astronomy and Astrophysics Program (AST-0702843 and AST0702821, respectively). G.T. acknowledges partial support from NASA grant NNX09AF59G. We acknowledge partial support also from the Kepler Mission under NASA Cooperative Agreement NCC2-1390 (D.W.L., PI). G.K. thanks the Hungarian Scientific Research Foundation (OTKA) for support through grant K-60750. L.L.K. is supported by the "Lendulet" Young Researchers Program of the Hungarian Academy of Sciences and the Hungarian OTKA grants K76816, K83790, and MB08C 81013. Tamás Szalai (University of Szeged) is acknowledged for his assistance during the ANU $2.3 \mathrm{~m}$ observations. This research has made use of Keck telescope time granted through NASA (N167Hr). This work is based in part on data collected at Subaru Telescope, which is operated by the National Astronomical Observatory of Japan, and in part on observations made with the Nordic Optical Telescope, operated on the island of La Palma jointly by Denmark, Finland, Iceland, Norway, and Sweden, in the Spanish Observatorio del Roque de los Muchachos of the Instituto de Astrofisica de Canarias. Thanks to G. Laughlin for useful advise on the Systemic Console, Dan Fabrycky and René Heller for useful comments, and special thanks to the anonymous referee for their helpful suggestions.

\section{REFERENCES}

Adams, F. C., \& Laughlin, G. 2006, ApJ, 649, 1004

Agol, E., Steffen, J., Sari, R., \& Clarkson, W. 2005, MNRAS, 359, 567

Bakos, G. Á., Koves, G., Torres, G., et al. 2007, ApJ, 670, 826

Bakos, G. Á., Noyes, R. W., Kovács, G., et al. 2004, PASP, 116, 266

Bakos, G. Á., Torres, G., Pál, A., et al. 2010, ApJ, 710, 1724
Béky, B., Bakos, G. Á., Hartman, J., et al. 2011, ApJ, 734, 109

Buchhave, L. A., Bakos, G. Á., Hartman, J. D., et al. 2011, ApJ, 733, 116

Butler, R. P., Marcy, G. W., Williams, E., et al. 1996, PASP, 108, 500

Carpenter, J. M. 2001, AJ, 121, 2851

Claret, A. 2004, A\&A, 428, 1001

Djupvik, A. A., \& Andersen, J. 2010, in Highlights in Spanish Astrophysics V, ed. J. M. Diego et al. (Berlin: Springer), 211

Droege, T. F., Richmond, M. W., \& Sallman, M. 2006, PASP, 118, 1666

Fogg, M. J., \& Nelson, R. P. 2007, A\&A, 461, 1195

Fürész, G. 2008, PhD thesis, Univ. Szeged, Hungary

Hansen, B. M. S., \& Barman, T. 2007, ApJ, 671, 861

Isaacson, H., \& Fischer, D. 2010, ApJ, 725, 875

Johnson, J. A., et al. 2011, ApJ, 735, 24

Kipping, D. M. 2009a, MNRAS, 392, 181

Kipping, D. M. 2009b, MNRAS, 396, 1797

Kipping, D. M. 2010, MNRAS, 407, 301

Kipping, D. M. 2011, PhD thesis, Univ. College London

Kipping, D. M., \& Bakos, G. A. 2011, ApJ, 730, 50

Kovács, G., Zucker, S., \& Mazeh, T. 2002, A\&A, 391, 369

Kovács, G., Bakos, G. Á., Hartman, J. D., et al. 2010, ApJ, 724, 866

Kovács, G., Bakos, G. Á., \& Noyes, R. W. 2005, MNRAS, 356, 557

Latham, D. W., Bakos, G. Á., Torres, G., et al. 2009, ApJ, 704, 1107

Liddle, A. R. 2007, MNRAS, 377, L74

Mandel, K., \& Agol, E. 2002, ApJ, 580, L171

Marcy, G. W., \& Butler, R. P. 1992, PASP, 104, 270

Meschiari, S., Wolf, A. S., Rivera, E., et al. 2009, PASP, 121, 1016

Noguchi, N., Aoki, K., Asai, R., et al. 2002, PASJ, 54, 819

Noyes, R. W., Hartmann, L. W., Baliunas, S. L., Duncan, D. K., \& Vaughan, A. H. 1984 , ApJ, 279,763

Pál, A., \& Bakos, G. Á. 2006, PASP, 118, 1474

Queloz, D., Henry, G. W., Sivan, J. P., et al. 2001, A\&A, 379, 279

Quinn, S. N., Bakos, G. Á., Hartman, J., et al. 2010, ApJ, submitted (arXiv:1008.3565)

Sato, B., Fischer, D. A., Henry, G. W., et al. 2005, ApJ, 633, 465

Sato, B., Kambe, E., Takeda, Y., Izumiura, H., \& Ando, H. 2002, PASJ, 54, 873

Schwarz, G. 1978, Ann. Stat., 6, 461

Seager, S., \& Mallén-Ornelas, G. 2003, ApJ, 585, 1083

Skrutskie, M. F., Cutri, R. M., Stiening, R., et al. 2006, AJ, 131, 1163

Tinetti, G., Vidal-Madjar, A., Liang, M.-C., et al. 2007, Nature, 448, 163

Torres, G., Bakos, G. Á., Kovcs, G., et al. 2007, ApJ, 666, 121

Valenti, J. A., \& Fischer, D. A. 2005, ApJS, 159, 141

Valenti, J. A., \& Piskunov, N. 1996, A\&AS, 118, 595

Vaughan, A. H., Preston, G. W., \& Wilson, O. C. 1978, PASP, 90, 267

Vogt, S. S., Allen, S. L., Bigelow, B. C., et al. 1994, Proc. SPIE, 2198, 362

Winn, J. N., Howard, A. W., Johnson, J. A., et al. 2011, AJ, 141, 63

Yi, S. K., Demarque, P., Kim, Y.-C., et al. 2001, ApJS, 136, 417 